\title{
Two Queues with Weighted One-Way Overflow
}

\section{Peter Sendfeld}

Published online: 28 February 2008

(C) Springer Science + Business Media, LLC 2008

\section{Erratum to: Methodol Comput Appl Probab DOI: 10.1007/s11009-007-9062-2}

In equation (13), (14), (38), (39), (53), (56), (61) and (67) the terms $(i+1) \wedge n_{1}$ and $(j+1) \wedge n_{2}$ should have been replaced with $\left((i+1) \wedge n_{1}\right)$ and $\left((j+1) \wedge n_{2}\right)$, respectively. They appear correctly below. This was a typographical error.

$$
\begin{gathered}
\left(\lambda_{1}\left(1-\delta_{i k_{1}}\right)\left(1-\chi_{i-n_{1}}\left(1-\chi_{j-n_{2}}\right)\right)+p \lambda_{1} \chi_{i-n_{1}}\left(1-\chi_{j-n_{2}}\right)\right) \\
\left.+\lambda_{2}\left(1-\delta_{j k_{2}}\right)+\left(i \wedge n_{1}\right) \mu_{1}+\left(j \wedge n_{2}\right) \mu_{2}\right) p_{i, j} \\
=\left(1-\chi_{i-n_{1}-1} \chi_{n_{2}-j-1}\right)\left(\lambda_{1}\left(1-\delta_{i 0}\right) p_{i-1, j}+\left(1-\delta_{j k_{2}}\right)\left((j+1) \wedge n_{2}\right) \mu_{2} p_{i, j+1}\right) \\
+\left(1-\delta_{j 0}\right)\left(p \lambda_{1} \delta_{i n_{1}} \chi_{n_{2}-j}+\lambda_{2}\left(1-\chi_{i-n_{1}-1} \chi_{n_{2}-j}\right)\right) p_{i, j-1} \\
+\left(1-\delta_{i k_{1}}\right)\left(\left(1-\chi_{i-n_{1}} \chi_{n_{2}-1-j}\right)\left((i+1) \wedge n_{1}\right) \mu_{1}+n_{2} \mu_{2} \delta_{j n_{2}} \chi_{i-n_{1}}\right) p_{i+1, j} \\
\left(\lambda_{1}+\lambda_{2}\left(1-\delta_{j k_{2}}\right)+i \mu_{1}+\left(j \wedge n_{2}\right) \mu_{2}\right) p_{i, j} \\
=\lambda_{1}\left(1-\delta_{i 0}\right) p_{i-1, j}+(i+1) \mu_{1} p_{i+1, j}+\left(1-\delta_{j 0}\right) \lambda_{2} p_{i, j-1} \\
+\left(1-\delta_{j k_{2}}\right)\left((j+1) \wedge n_{2}\right) \mu_{2} p_{i, j+1} .
\end{gathered}
$$

The online version of the original article can be found at http://dx.doi.org/10.1007/s11009-007-9062-2

P. Sendfeld $(\bowtie)$

Department of Mathematics and Computer Science,

University of Osnabrück, Osnabrück, Germany

e-mail: peter.sendfeld@math.uni-osnabrueck.de 


$$
\begin{aligned}
\left(\lambda_{1}\left(1-\delta_{i k_{1}} \chi_{j-n_{2}}\right)\left(1-\chi_{i-n_{1}}\left(1-\chi_{j-n_{2}}\right)\right)+p \lambda_{1} \chi_{i-n_{1}}\left(1-\chi_{j-n_{2}}\right)\right) \\
\left.+\lambda_{2}\left(1-\delta_{j k_{2}}\right)+\left(i \wedge n_{1}\right) \mu_{1}+\left(j \wedge n_{2}\right) \mu_{2}\right) p_{i, j} \\
=\left(1-\chi_{i-n_{1}-1} \chi_{n_{2}-j-1}\right)\left(\lambda_{1}\left(1-\delta_{i 0}\right) p_{i-1, j}\right)+\left(1-\delta_{i k_{1}}\right)\left((i+1) \wedge n_{1}\right) \mu_{1} p_{i+1, j} \\
\quad+\left(1-\delta_{j 0}\right)\left(p \lambda_{1} \chi_{i-n_{1}} \chi_{n_{2}-j}+\lambda_{2}\right) p_{i, j-1}+\left(1-\delta_{j k_{2}}\right)\left((j+1) \wedge n_{2}\right) \mu_{2} p_{i, j+1}
\end{aligned}
$$

$$
\begin{aligned}
& \left(\lambda_{1}\left(1-\delta_{i k_{1}}\right)\left(1-\chi_{i-n_{1}}\left(1-\chi_{j-n_{2}}\right)\right)+p \lambda_{1} \chi_{i-n_{1}}\left(1-\chi_{j-n_{2}}\right)\right) \\
& \left.+\lambda_{2}\left(1-\delta_{j k_{2}}\right)+\left(i \wedge n_{1}\right) \mu_{1}+\left(j \wedge n_{2}\right) \mu_{2}\right) p_{i, j} \\
& =\left(1-\chi_{i-n_{1}-1}\left(1-\delta_{j k_{2}}\right)\right)\left(\lambda_{1}\left(1-\delta_{i 0}\right) p_{i-1, j}+\left(1-\delta_{j k_{2}}\right)\left((j+1) \wedge n_{2}\right) \mu_{2} p_{i, j+1}\right) \\
& \quad+\left(1-\delta_{j 0}\right)\left(p \lambda_{1} \delta_{i n_{1}} \chi_{n_{2}-j}+\lambda_{1} \delta_{i n_{1}}\left(1-\chi_{n_{2}-j}\right)+\lambda_{2}\left(1-\chi_{i-n_{1}-1}\right)\right) p_{i, j-1} \\
& \quad+\left(1-\delta_{i k_{1}}\right)\left(\left(1-\chi_{i-n_{1}}\left(1-\delta_{j k_{2}}\right)\right)\left((i+1) \wedge n_{1}\right) \mu_{1}+n_{2} \mu_{2} \delta_{j n_{2}} \chi_{i-n_{1}}\right) p_{i+1, j} \\
& \quad \\
& \quad\left(\lambda_{1}\left(1-\delta_{i k_{1}} \delta_{j k_{2}}\right)+\lambda_{2}\left(1-\delta_{j k_{2}}\right)+\left(i \wedge n_{1}\right) \mu_{1}+\left(j \wedge n_{2}\right) \mu_{2}\right) p_{i, j} \\
& \quad=\left(1-\delta_{i 0}\right)\left(1-\chi_{i-n_{1}-1} \delta_{j k_{2}}\right) \lambda_{1} p_{i-1, j}+\left(1-\delta_{i k_{1}}\right)\left((i+1) \wedge n_{1}\right) \mu_{1} p_{i+1, j} \\
& \quad+\left(1-\delta_{j 0}\right)\left(\lambda_{1} \chi_{i-n_{1}}+\lambda_{2}\right) p_{i, j-1}+\left(1-\delta_{j k_{2}}\right)\left((j+1) \wedge n_{2}\right) \mu_{2} p_{i, j+1}
\end{aligned}
$$

$$
\begin{aligned}
\left(\lambda_{1}(1-\right. & \left.\delta_{i k_{1}}\right)\left(1-\chi_{i-n_{1}}\left(1-\delta_{j k_{2}}\right)\right)+p \lambda_{1} \chi_{i-n_{1}}\left(1-\delta_{j k_{2}}\right) \\
+ & \left.\lambda_{2}\left(1-\delta_{j k_{2}}\right)+\left(i \wedge n_{1}\right) \mu_{1}+\left(j \wedge n_{2}\right) \mu_{2}\right) p_{i, j} \\
= & \left(1-\chi_{i-n_{1}-1}\left(1-\delta_{j k_{2}}\right)\right)\left(\lambda_{1}\left(1-\delta_{i 0}\right) p_{i-1, j}+\left(1-\delta_{j k_{2}}\right)\left((j+1) \wedge n_{2}\right) \mu_{2} p_{i, j+1}\right) \\
& +\left(1-\delta_{j 0}\right)\left(p \lambda_{1} \delta_{i n_{1}}+\lambda_{2}\left(1-\chi_{i-n_{1}-1}\right)\right) p_{i, j-1} \\
& +\left(1-\delta_{i k_{1}}\right)\left(\left(1-\chi_{i-n_{1}}\left(1-\delta_{j k_{2}}\right)\right)\left((i+1) \wedge n_{1}\right) \mu_{1}+n_{2} \mu_{2} \delta_{j k_{2}} \chi_{i-n_{1}}\right) p_{i+1, j}
\end{aligned}
$$

$$
\begin{aligned}
\left(\lambda_{1}\left(1-\delta_{i k_{1}}\right)\left(1-\delta_{i k_{1}}\left(1-\chi_{j-n_{2}}\right)\right)+p \lambda_{1} \delta_{i k_{1}}\left(1-\chi_{j-n_{2}}\right)+\lambda_{2}\left(1-\delta_{j k_{2}}\right)\right. \\
\left.\quad+\left(i \wedge n_{1}\right) \mu_{1}+\left(j \wedge n_{2}\right) \mu_{2}\right) p_{i, j} \\
=\lambda_{1}\left(1-\delta_{i 0}\right) p_{i-1, j}+\left(1-\delta_{i k_{1}}\right)\left((i+1) \wedge n_{1}\right) \mu_{1} p_{i+1, j} \\
\quad+\left(1-\delta_{j 0}\right)\left(p \lambda_{1} \delta_{i k_{1}} \chi_{n_{2}-j}+\lambda_{2}\right) p_{i, j-1}+\left(1-\delta_{j k_{2}}\right)\left((j+1) \wedge n_{2}\right) \mu_{2} p_{i, j+1}
\end{aligned}
$$

$$
\begin{gathered}
\left(\lambda_{1}\left(1-\delta_{i k_{1}}\right)+p \lambda_{1} \delta_{i k_{1}}\left(1-\delta_{j k_{2}}\right)+\lambda_{2}\left(1-\delta_{j k_{2}}\right)+\left(i \wedge n_{1}\right) \mu_{1}+\left(j \wedge n_{2}\right) \mu_{2}\right) p_{i, j} \\
=\lambda_{1}\left(1-\delta_{i 0}\right) p_{i-1, j}+\left(1-\delta_{i k_{1}}\right)\left((i+1) \wedge n_{1}\right) \mu_{1} p_{i+1, j} \\
\quad+\left(1-\delta_{j 0}\right)\left(p \lambda_{1} \delta_{i k_{1}}+\lambda_{2}\right) p_{i, j-1}+\left(1-\delta_{j k_{2}}\right)\left((j+1) \wedge n_{2}\right) \mu_{2} p_{i, j+1}
\end{gathered}
$$

